\title{
A Unique Coexistence of Rectal Adenocarcinoma and Gastric Antral Gastrointestinal Stromal Tumor: A Case Report and Minireview
}

\author{
Mahmoud Rezk-Abdelwahed Hussein ${ }^{\mathrm{a}}$, Abdullah Saad Alqahtani ${ }^{\mathrm{b}}$, h, Ahmed Mostafa Mohamed ${ }^{\mathrm{c}}$, \\ Yahia Ibraheem Assiri ${ }^{\mathrm{d}}$, Nasser Ibraheem Alqahtani ${ }^{\mathrm{e}}$, Saeed Ali Alqahtani ${ }^{\mathrm{b}}$, \\ Hatim Mahgoub Alic, Asim Abdallah Ahmed Elyas ${ }^{\mathrm{b}}$, Mubarak Mohammed Al-Shraim ${ }^{\mathrm{f}}$, \\ Syed Shamshad Hussain ${ }^{\text {, }}$ Eman E. Abu-Dief ${ }^{\mathrm{g}}$
}

\begin{abstract}
Several studies have reported the coexistence of gastric gastrointestinal stromal tumors (GISTs) with many primary carcinomas such as gastric and renal cell carcinomas. However, to date reports about the coexistence of gastric GISTs and colorectal adenocarcinoma are limited. Herein we report a unique case of gastric GIST coexisting synchronously with rectal adenocarcinoma in a 36-year-old male patient who presented with weight loss, vomiting, and bleeding per rectum. Computed tomography (CT) revealed circumferential rectal mass coexistent with an irregular gastric soft tissue mass. The diagnosis of rectal adenocarcinoma and gastric GIST was established by immunohistological evaluation of the colonoscopic (rectum) and CT-guided (stomach) biopsies. The patient received concomitant chemoradiotherapy for the rectal adenocarcinoma and neoadjuvant imatinib for the gastric GIST. This was followed by low anterior resection with total mesorectal excision and wedge resection of the gastric mass. Follow-up of the patient for 1.5 years revealed no evidence of disease recurrence. We also present a minireview of the literature that provides insights into this subject as a separate section.
\end{abstract}

Manuscript submitted November 9, 2021, accepted December 4, 2021

Published online December 13, 2021

aDepartment of Pathology, Faculty of Medicine, Assiut University, Assiut, Egypt bDepartment of General Surgery, Armed Forces Hospital, Southern Region, Saudi Arabia

'Department of Oncology, Armed Forces Hospital, Southern Region, Saudi Arabia

${ }^{\mathrm{d} D e p a r t m e n t ~ o f ~ R a d i o l o g y, ~ C o l l e g e ~ o f ~ M e d i c i n e, ~ K i n g ~ K h a l i d ~ U n i v e r s i t y, ~ A b h a, ~}$ Saudi Arabia

eDepartment of Gastroenterology, Armed Forces Hospital, Southern Region, Saudi Arabia

fDepartment of Pathology, College of Medicine, King Khalid University, Abha, Saudi Arabia

gDepartment of Histology, Faculty of Medicine, Sohag and Merit Universities, Sohag, Egypt

${ }^{\mathrm{h}}$ Corresponding Author: Abdullah Saad Alqahtani, Department of General Surgery, Armed Forces Hospital, Southern Region, Saudi Arabia. Email: dr_ asq@yahoo.com

doi: https://doi.org/10.14740/gr1482
Keywords: GIST; Stomach; Carcinoma; Rectum; KIT; Stem cell factor

\section{Introduction}

\section{An overview of the gastrointestinal stromal tumors (GISTs)}

Gastrointestinal stromal tumors (GISTs) are the most common mesenchymal tumors of the gastrointestinal tract with the stomach, and the small intestine (ileum and jejunum) being the most common site [1]. In the stomach, the body is the most common site of the GISTs (70\%) followed by the cardia or the antrum (15\% each). In 1983, Mazur et al introduced the term "gastrointestinal stromal tumors, GISTs" to lump a group of mesenchymal tumors with neurogenic or myogenic histological features but lacking the immunohistochemical characteristics of the Schwann cells and the ultra structural findings of the smooth muscle cells [2]. Initial thoughts considered GISTs as smooth muscle neoplasms and therefore these tumors were labeled as gastrointestinal smooth muscle tumors, leiomyomas, leiomyoblastomas, leiomyosarcomas, or smooth muscle tumors of uncertain malignant potential. Other thoughts regarded GISTs as neurogenic tumors and as such, they were called gastrointestinal autonomic nerve tumors and gastrointestinal pacemaker cell tumors $[3,4]$.

\section{The role of C-KIT tyrosine kinase receptor in GIST}

In 1986, the Hardy-Zuckerman 4 feline sarcoma retrovirus was first isolated from the feline fibrosarcoma. Its viral genome contained a new oncogene that was coined as $v$-KIT, which encoded a transmembrane tyrosine kinase receptor called "KIT". Its cellular homolog is C-KIT. Several studies indicated that mice with mutations in the $C$-KIT gene lacked the network of interstitial cells of Cajal associated with the intestinal pacemaker and Auerbach's nerve plexus [3-5]. In 1998, GIST was reliably separated from the other mesenchymal tumors follow- 
ing the discovery of the gain-of-function mutations in the $C-K I T$ proto-oncogene [6]. A growing body of evidence indicates that GIST derives from the interstitial cells of Cajal or their multipotential mesenchymal precursor cells. The oncogenic mutations of the receptor tyrosine kinase (RTK) proto-oncogenes C-KIT and the platelet-derived growth factor receptor alpha $(P D G-$ $F R A$ ) gene are involved in the development of most cases of GISTs [6-8]. These mutations lead to the phosphorylation of the RTKs. Protein kinase (PK) can catalyze the transfer of phosphate, diphosphate, nucleotide residues, and other groups to the receptor molecules. The phosphorylation of RTKs results in the stimulation of the downstream pathways leading to enhanced cell proliferation and survival. The other molecular events that trigger GISTs include alterations of the succinate dehydrogenase [9], and mutations of $N F 1$, and RAS/RAF/MEK pathway [6-8]. C-KIT (a proto-oncogene) is RTK and its ligand is a stem cell factor (SCF) which is a cytokine having several important roles in the development of the precursor cells $[10,11]$.

\section{The expression of C-KIT tyrosine kinase receptor and its ligand SCF in colorectal cancer (CRC)}

$\mathrm{CRC}$ is a common cause of cancer that affects both males and females worldwide. Several molecular pathways are involved in the development of $\mathrm{CRC}$ such as chromosomal alterations, and microsatellite instability. $\mathrm{CRC}$ is characterized by mutations that activate RTKs, and therefore it is conceivable that these cancers are sensitive to the therapeutic inhibition strategies that target RTKs [12]. In GISTs, the activation of C-KIT following the binding with SCF triggers several signaling pathways including ones that have important roles in the pathogenesis of CRC such as the JAK/STAT, PI3K, and RAS pathways [10, 11].

The expression of C-KIT and SCF in CRC was previously reported by some studies [13-16]. Sammarco et al examined tissue specimens of CRC from 20 patients using the immunohistochemical and molecular methods. C-KIT protein expression was positive in the interstitial cells of Cajal whereas it was absent in normal colonic epithelium. In the cell culture model (semisolid medium), the exogenous supplementation with SCF helps the anchorage-independent growth of HT-29 colon carcinoma cells [17]. In colorectal mucinous carcinomas, C-KIT was absent except in one case of poorly differentiated adenocarcinoma in which $\mathrm{C}$-KIT protein expression was identified in the malignant epithelium [14].

In accordance with data from The Cancer Genome Atlas [18], Chen and his colleagues reported C-KIT protein expression in tumor cells of $51 \%$ of the colorectal carcinomas [15]. Fatrai et al indicated that colon carcinomas contain some undifferentiated stem cell-like cancer cells that have high tumorigenic potential. The authors used the undifferentiated colonosphere cultures from human colon tumors to propagate stably differentiated cell lines. SCF cytokine was released by the differentiated tumor cells and they were able to support the clonogenic capacity of KIT-positive colonosphere cells. The inhibition of KIT or SCF prevented the epithelial to mesenchymal transition in colonosperes. The clonogenic activity can be blocked by both anti-SCF and C-KIT inhibitors [16]. The stem cell-like features of tumor-initiating cells that support CRC cell renewal and metastasis are C-KIT or SCF positive and may represent therapeutic targets [16].

Lahm et al investigated SCF production in the cell culture models of CRC. They reported C-KIT expression in two cell lines (LS174T and LS1034) and SCF in nine of 11 tested CRC cell lines. They also indicated that a single SCF mRNA transcript was seen (Northern blot) and two lines (LS174T and HT29) synthesized SCF protein. SCF was found to stimulate the proliferation and colony formation of LS174T cell line. Also, C-KIT protein was expressed in LS174T cells and a neutralizing anti-C-KIT monoclonal antibody $(\mathrm{mAb})$ inhibited colony formation of LS174T [19].

\section{Coexistence of CRC and GIST}

There is an increasing number of reports indicating the coexistence of GIST with synchronous and metachronous neoplasms of histologically diverse origins. They include gastrointestinal tumors (adenocarcinomas, neuroendocrine tumors, and schwannomas) genitourinary carcinomas (urothelial, prostatic, renal, adrenal, and ovarian carcinomas), lung carcinomas (squamous cell and small cell carcinomas), skin malignancies (melanomas), peritoneal tumors (mesotheliomas), lymphomas, and soft tissue sarcomas (Ewing's sarcoma, dermatofibrosaracoma) $[8,20]$. Vassos et al examined the clinicopathologic features of 30 cases with GISTs over 10 years. They indicated that tumors associated with GIST included a variety of hemato-lymphoid, renal, gastrointestinal, urological, skin, and thyroid follicular neoplasms [8].

Although several case studies of coexistent GISTs synchronous or metachronous with other tumors have been documented in the literature $[8,20]$, to date only a few cases of gastric GISTs synchronous with rectal adenocarcinomas have been reported [21-26]. A summary of these reports is presented in Table 1 [21-27].

\section{Case Report}

Herein, we report our experience with a single case of a gastric antral GIST coexisting synchronously with adenocarcinomas of the rectum. The clinical, endoscopic, and radiological features of the case were discussed. The relevant studies were discussed. Informed consent was obtained from the patient.

\section{Colonoscopic, radiological and histological features of the rectal mass}

A36-year-old medically free male patient was referred to the Department of General Surgery for a recent complaining of loss of weight, nausea, vomiting, and bleeding per rectum. Lower gastrointestinal endoscopy was done and revealed a circumferential ugly-looking mass just above the anal verge about $9 \mathrm{~cm}$ in length and multiple punch biopsies were obtained from the lesion. The histological sections from the rectal mass re- 
Table 1. Summary of the Previous Studies About Synchronous Colorectal Carcinoma and Other Malignant Neoplasms

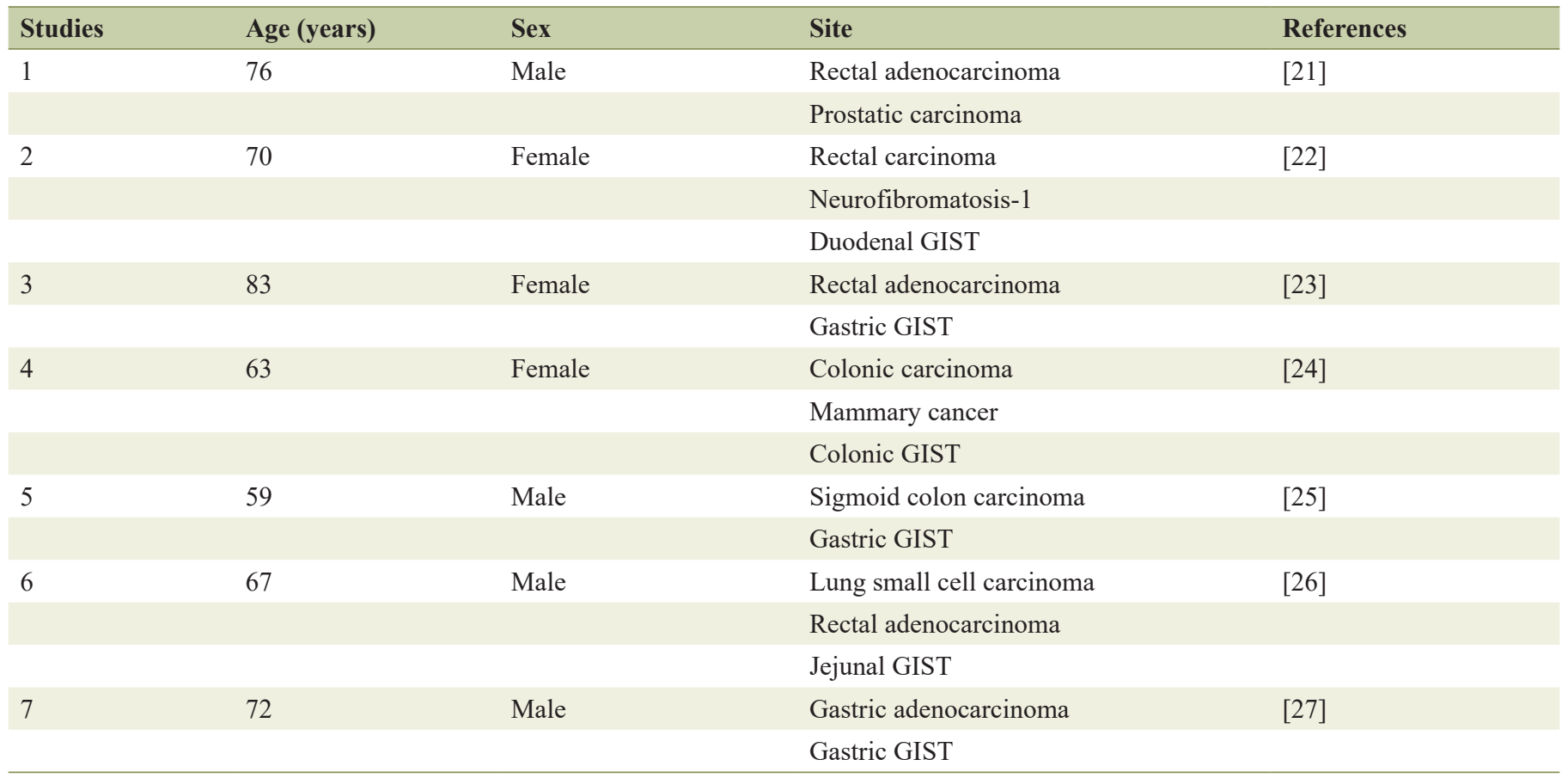

GIST: gastric gastrointestinal stromal tumor.

vealed features of poorly differentiated adenocarcinoma (malignant cells arranged in complicated glandular patterns amid desmoplastic stroma). The malignant cells were positive for pancytokeratin, cytokeratin (CK) 20, and p53 whereas staining for cluster of differentiation (CD) 117 (C-KIT protein), DOG-1(discovered on GIST-1 protein), CD34, S100, desmin, HMB45, and smooth muscle actin was negative.

Baseline computed tomography (CT) chest abdomen pelvis (CAP) examination was done on March 25, 2020. It revealed an irregular thickening of the rectal wall which started about $4 \mathrm{~cm}$ from the anal verge and extended upward for roughly $9 \mathrm{~cm}$ associated with stranding of the perirectal fat with a small few enlarged lymph nodes largest measuring $1 \times$ $0.8 \mathrm{~cm}$. There was no evidence of distant metastases. The magnetic resonance imaging (MRI) pelvis was performed on April 20,2020 for the further staging of the rectal mass. It revealed a rectal mass arising $4.3 \mathrm{~cm}$ from the anal verge and extending cranially up to $9 \mathrm{~cm}$. The lesion had a maximum extension of approximately $0.8 \mathrm{~cm}$ beyond the muscularis propria denoting the T3c lesion. This lesion was associated with extensive perirectal fat stranding and edema. There were multiple abnormally enlarged internal iliac lymph nodes and the largest measuring around $0.8 \mathrm{~cm}$ with irregular capsule and heterogeneous signal intensity. A summary of the colonoscopic, histological, and radiological findings are shown in Figures 1, 2.

\section{Radiological and histological features of the gastric antral mass}

Baseline CAP CT examination was done on March 25, 2020. It revealed an irregular soft tissue lesion was arising from the anterior aspect of the gastric antrum, at the anterior aspect of the right side of the peritoneal cavity anterior to the transverse colon measuring $7.2 \times 3.8 \times 6.6 \mathrm{~cm}$. The lesion had mass effects on the transverse colon, indenting and displacing the colon posteriorly. CT-guided biopsies were obtained from the gastric antral mass. On histology, sections revealed a malignant neoplasm composed of mitotically active, mildly pleomorphic epithelioid cells with abundant pink cytoplasm admixed with some shortly spindly cells with vesicular nuclei. The mitotic activity was 3/50 high-power field (HPF) and the risk stratification was low to moderate. The tumor cells were positive for CD117, DOG-1, and CD34 whereas staining for pancytokeratin (AE1/AE3), CK20, CK7, CDX2, CD31, smooth muscle actin, desmin, HMB45, and S100 was negative. Ki67 proliferation index was low (approximately 2-3\%). A summary of immunohistological findings are shown in Figure 3.

\section{Preoperative chemotherapy and surgical intervention}

Based on the baseline radiological and histopathological findings, the gastric tumor was staged as cT1b N0 M0 gastric GIST whereas the rectal tumor was staged as cT3c N2 M0. The case was discussed in the tumor board in April 2020 and the plan was to offer the patient neoadjuvant concomitant chemoradiotherapy for rectal cancer and neoadjuvant imatinib for gastric GIST. The patient was started on imatinib (400 mg once daily) in April 2020. The patient was started on rectal concurrent radiochemotherapy (CCRT) in May 2020 using conventional fractionation $180 \mathrm{cGy} /$ fraction for 28 fractions 

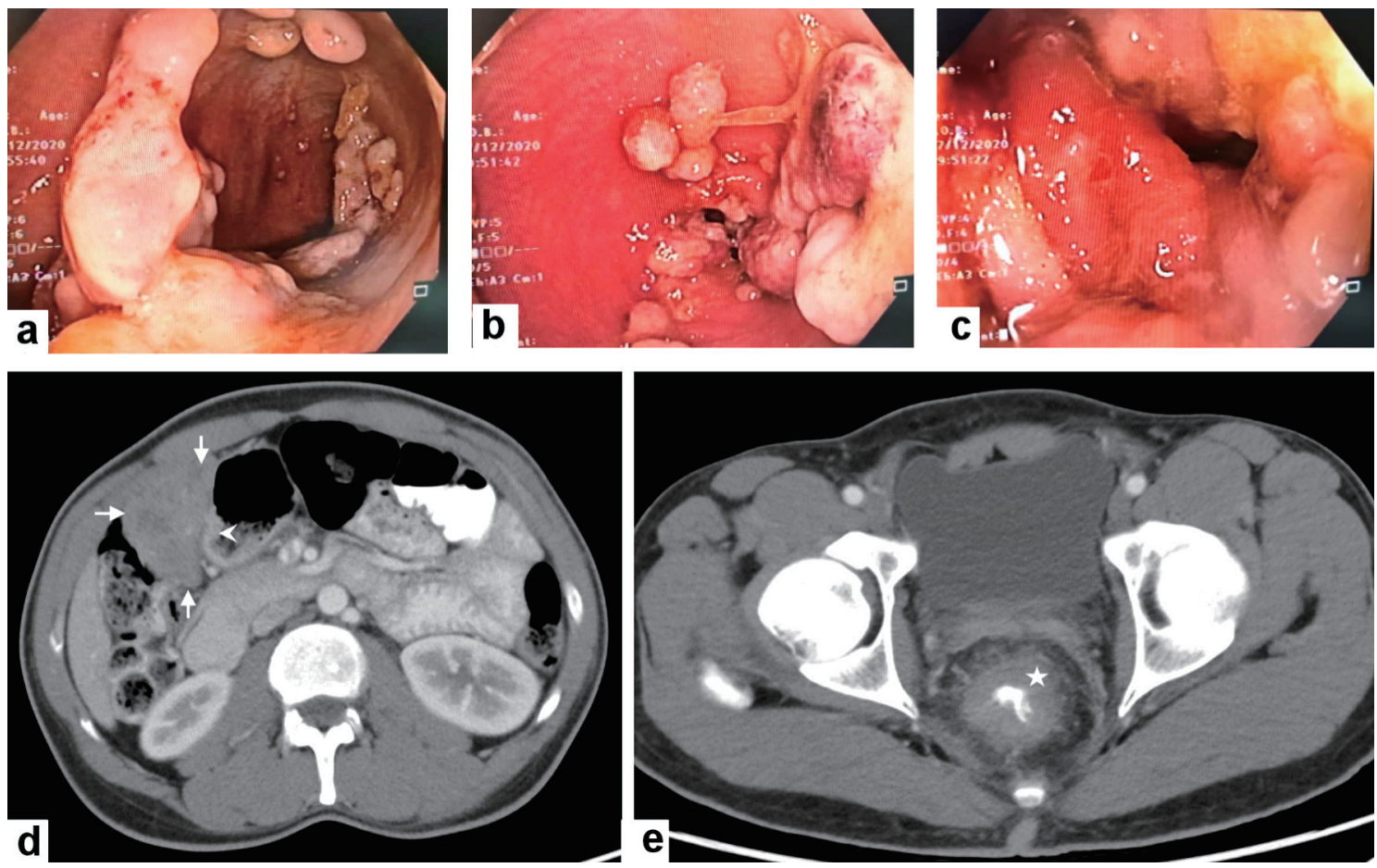

Figure 1. Colonoscopic and radiological features of the gastric GIST and rectal adenocarcinoma. (a, b, c) Colonoscopy revealed the presence of a circumferential ugly-looking rectal fungating mass. (d) Contrast-enhanced computed tomography (CECT) scan of the abdomen reveals a large exophytic enhancing mass arising (arrows) from gastric pylorus (arrowhead) likely GIST. (e) CECT scan through the pelvis shows a circumferential wall thickening of the low rectum (asterisk). GIST: gastric gastrointestinal stromal tumor.
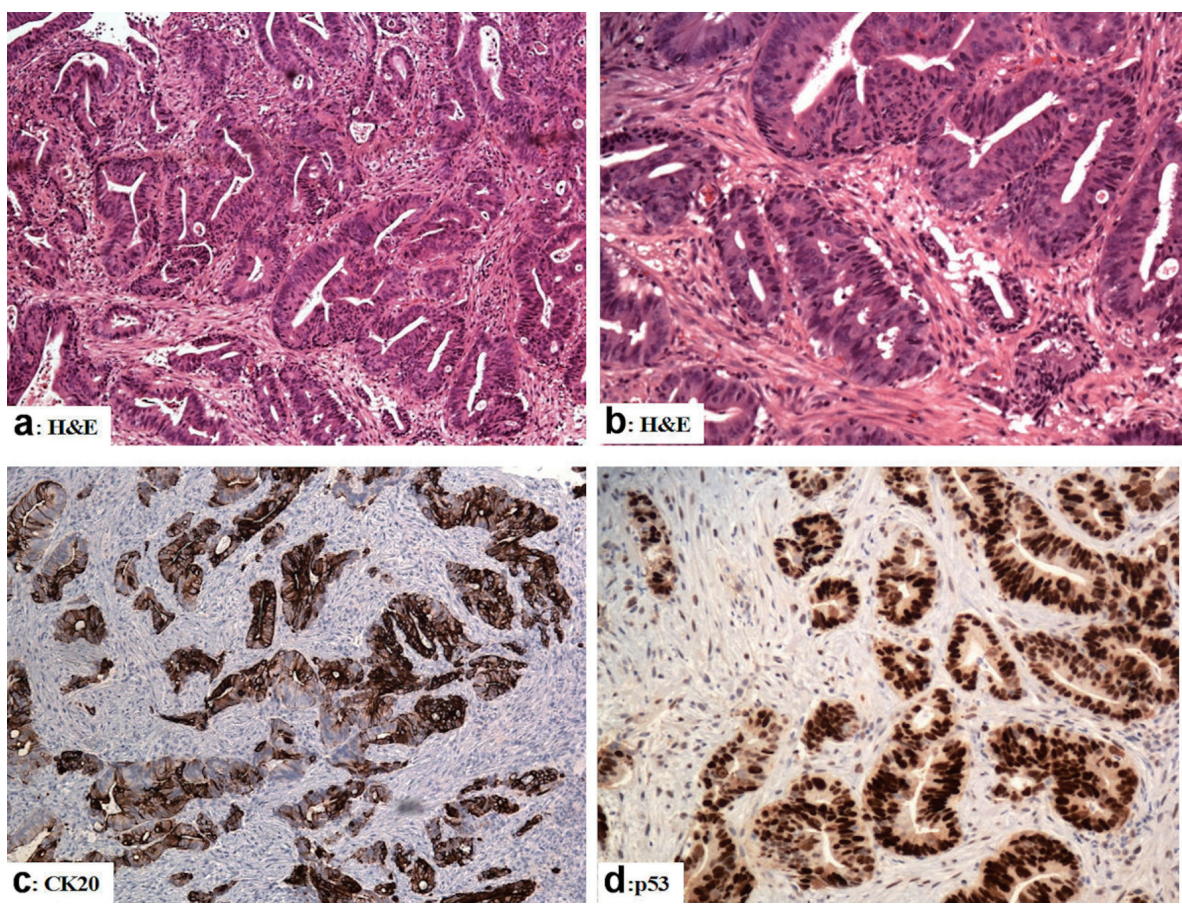

Figure 2. The immunohistological features of the rectal adenocarcinoma (colonoscopic biopsies). (a, b) Sections from the rectal mass show malignant glands with a complicated glandular pattern haphazardly scattered amid the desmoplastic stroma (H\&E). (c) The tumor cells are reactive for CK20. (d) Mutation-type p53 diffuse strong reactivity in the tumor cells. Original magnifications: $(a) \times 100,(b) \times 200,(c) \times 100,(d) \times 200$. H\&E: hematoxylin and eosin stain; CK: cytokeratin. 

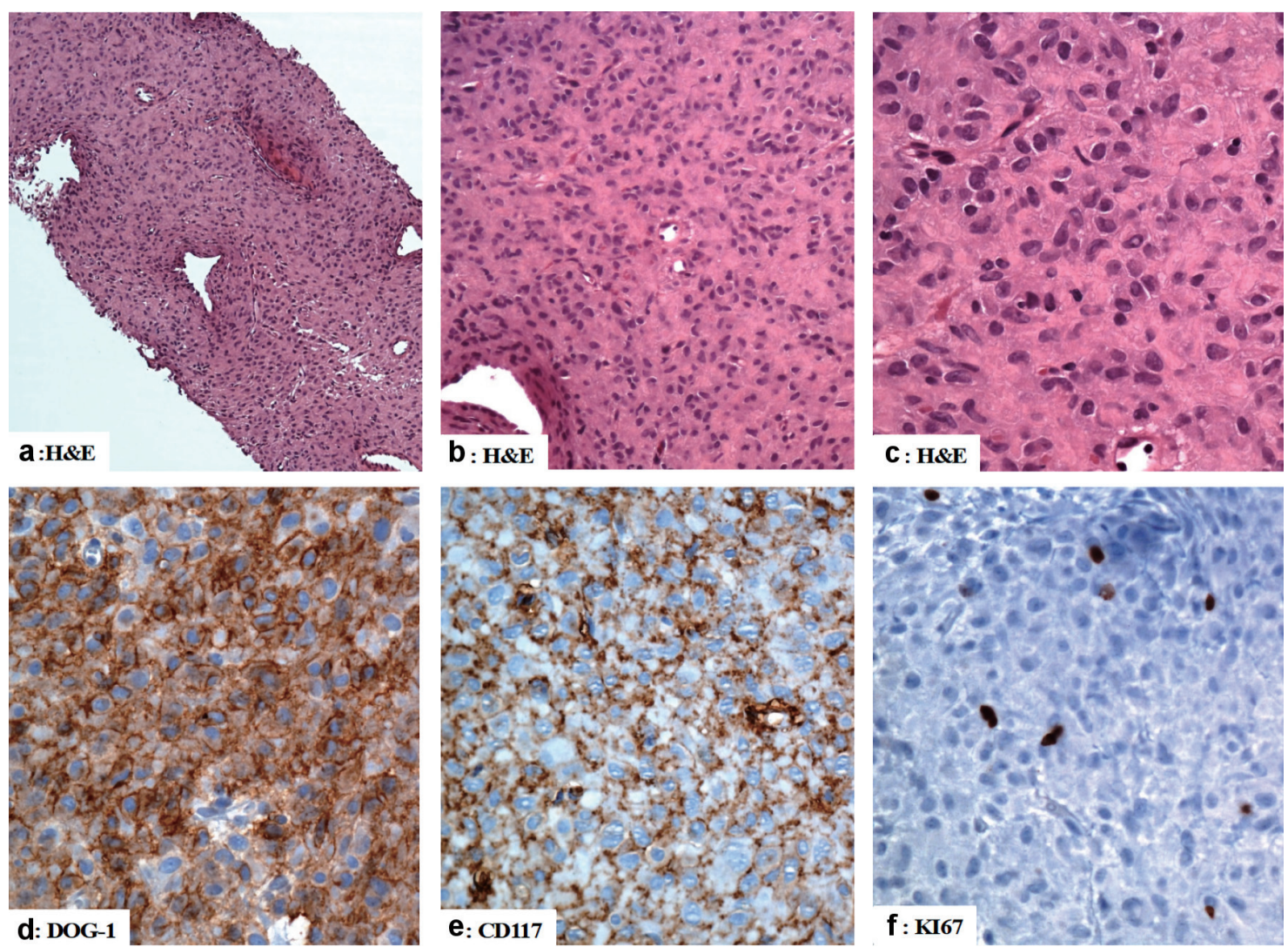

Figure 3. The immunohistological features of the gastric gastrointestinal stromal tumor (computed tomography (CT)-guided biopsies). ( $a, b, c)$ The tumor is composed of mildly pleomorphic atypical spindle and epithelioid-shaped cells with eosinophilic cytoplasm arranged in a solid pattern. (d, e) The tumor cells are positive for DOG-1, and CD117. (f) The Ki67 labeling index is low. Original magnifications: (a) $\times 40$, (b) $\times 200$, (c) $\times 400,(d) \times 400,(e) \times 400$, and (f) $\times 400$. H\&E: hematoxylin and eosin stain; CD: cluster of differentiation.

total dose $(5,040 \mathrm{cGy})$ concomitantly with oral capecitabine $\left(825 \mathrm{mg} / \mathrm{m}^{2}\right.$ twice daily/5 days per week). Complete blood count, liver function tests, and kidney function tests were requested regularly every 2 weeks during the treatment course. The treatment-related adverse effects during the course of radiation were within accepted ranges and no grade 3 adverse effects were reported. The reported grade 1 and 2 treatmentrelated adverse effects included the following: anemia, neutropenia, diarrhea, nausea, vomiting, and dry desquamation of the skin. After completing the course of the neoadjuvant therapy restaging CT CAP was performed on July 30, 2020. The findings were compared to the CT CAP done on March 25, 2020 and it indicated a nearly complete resolution of the previously seen right side abdominal mass indicating a good response to treatment. However, there were no obvious changes in the rectal mass denoting stationary response. These findings were confirmed by MRI pelvis.

\section{Chemotherapy and follow-up of the patient}

The preoperative neoadjuvant radiotherapy and chemotherapy were followed by low anterior resection with total mesorectal excision and ultra-low colorectal anastomosis $3-4 \mathrm{~cm}$ proximal to anal verge with diverting temporary loop transverse colostomy that was closed later on. A wedge resection of gastric GIST was also done in the same setting (September 2020). Gross examination of the rectal specimen revealed a focally congested and edematous mucosa. No masses or ulcers were seen. Histologically, there was no residual tumor and the lymph nodes in the perirectal fatty tissues were negative for malignancy, i.e., complete pathological response within the primary tumor and the dissected lymph nodes. Gross examination of the gastric antral specimen revealed a residual disease in the form of $6.0 \times 5.0 \times 2.5 \mathrm{~cm}$ firm mass with a grey cut section. Histologically, sections from the mass confirmed the diagnosis of GIST (epithelioid cell type). The resection margins were negative for neoplastic cells. The tumor cells were reactive for CD117, DOG-1, and CD34 and negative for pancytokeratin (AE1/AE3), CK20, CK7, CDX2, CD31, smooth muscle actin, desmin, HMB45, and S100. The mitotic count was four mitoses per $50 \mathrm{HPFs}$, and the Ki67 proliferation index was approximately $10 \%$. The risk stratification was low to moderate. A summary of the histological findings are shown in Figure 4.

In October 2020 the patient started his adjuvant treatment using CAPOX protocol (oxaliplatin $130 \mathrm{mg} / \mathrm{m}^{2}$ and capecitabine $1,000 \mathrm{mg} / \mathrm{m}^{2}$ cycled every 3 weeks) concomitantly with 

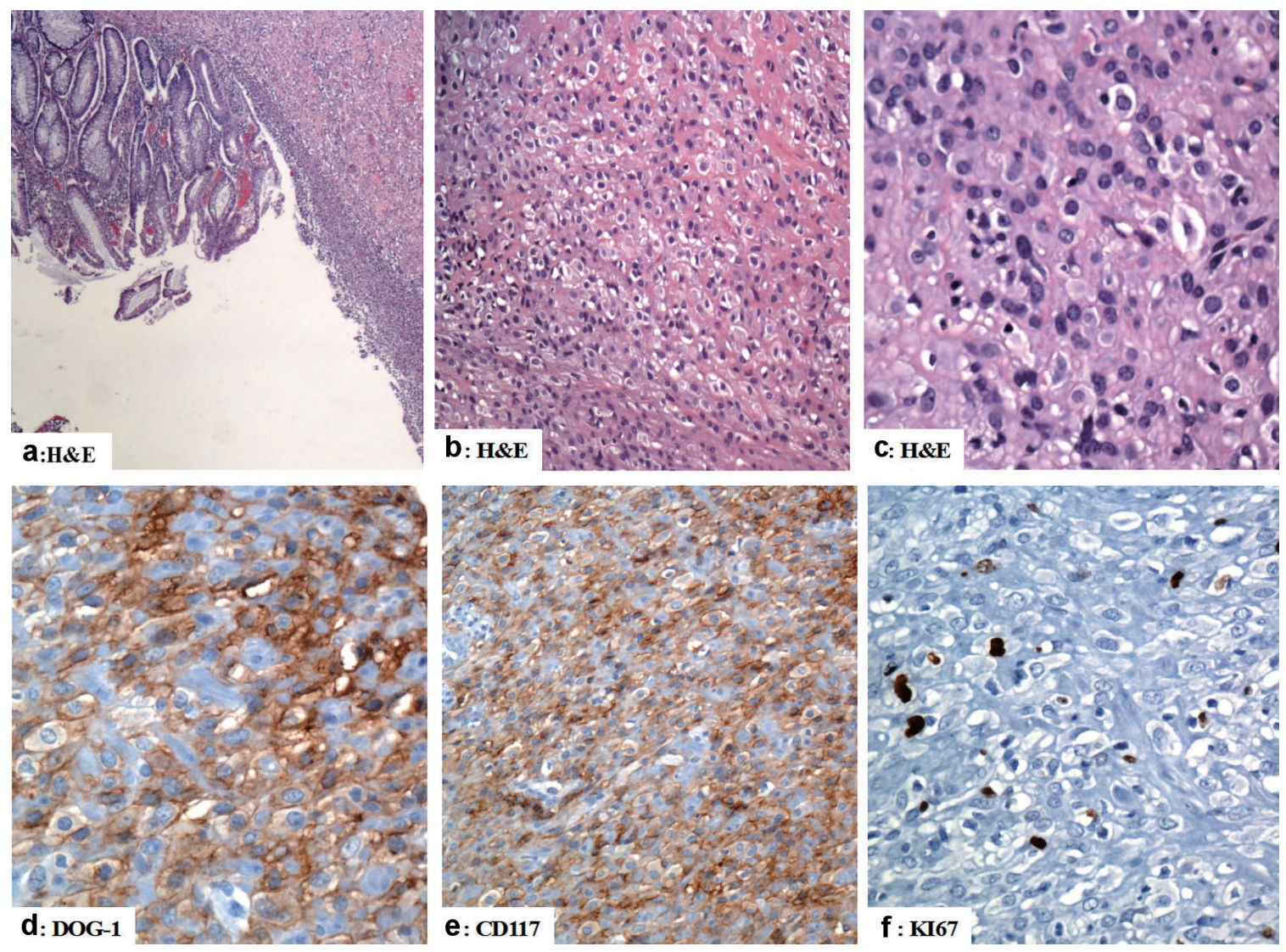

Figure 4. The immunohistological features of the gastric gastrointestinal stromal tumor (excision of the gastric GIST). (a) There is focal ulceration of the mucosa with subepithelial neoplastic cells arranged in sheets. (b, c) The tumor cells have epithelioid morphology with vesicular nuclei and pink cytoplasm. (d, e) The tumor cells are positive for DOG-1 and CD117 (d and e, respectively). (f) Ki67 labeling index is low (less than 5\%). Original magnifications: (a) $\times 40$, (b) $\times 200$, (c) $\times 400$, (d) $\times 400,(e) \times 200$ and (f) $\times 400$. H\&E: hematoxylin and eosin stain; CD: cluster of differentiation.

imatinib $300 \mathrm{mg}$ per os $(\mathrm{PO})$ once daily. We have started with a reduced dose to evaluate the tolerability of the patient to the treatment protocols. Imatinib dose was then increased after 2 months to $400 \mathrm{mg}$ once daily as there was no reporting of grade 3 or more adverse effects (specifically the hematological toxicity). After six cycles of CAPOX, oxaliplatin was discontinued as the patient developed grade 3 neurotoxicity and he continued treatment for two more cycles using capecitabine single agent and the last cycle was given on April 2021. Complete blood picture, liver function tests, and renal function tests were requested on regular basis before each cycle of CAPOX and capecitabine therapy.

The patient developed grade 3 neutropenia before the last cycle of capecitabine, and treatment was therefore delayed for 1 week with coadministration of granulocyte colony-stimulating factor (C-GSF) till the recovery of neutrophil count. Grade 1 and 2 adverse effects that were reported during the adjuvant setting included the following: anemia, neutropenia, diarrhea, and oral mucositis. The plan for the patient was to continue adjuvant imatinib for 1 year postoperatively. In December, 2021, a follow-up of the patient (clinical examination, laboratory, and radiological investigations) revealed that the patient has been doing very well with no evidence of disease recurrence.
Genetic counseling was provided to the patient and his family relatives.

\section{Discussion}

An increasing body of evidence indicates the concurrent occurrence of GISTs and other neoplasms such as carcinomas of the digestive tract, breast, neuroendocrine, liver, urogenital system, and hemato-lymphoid malignancies $[8,20]$. Here we report a case of a gastric antral GIST occurring synchronously with poorly differentiated adenocarcinomas of the rectum. To the best of our knowledge, only a few cases of concurrent gastric antral GISTs and rectal adenocarcinoma were reported to date. The unique aspect of the case reported by our study is the exceptionally rare coexistence of GIST with CRC in a young patient.

The clinicopathologic features of the case presented here concur with previous studies [21-28]. Zhang et al examined 32 cases of concurrent GIST and carcinoma of the gastrointestinal tract [28]. The stomach was the most common site of carcinomas (concurrent with GIST) followed by the esophagus, duodenum, and colorectum. Bellone et al examined the roles of 
the $\mathrm{C}-\mathrm{KIT} / \mathrm{SCF}$ in the development of the infiltrative properties of DLD-1 colonic carcinoma cells in vitro cell culture model. The authors indicated that SCF was important not only for the migration of carcinoma cells but also for their invasion through the reconstituted basement membranes (Matrigel). SCF was able to upregulate the expression of matrix metalloproteinase- 9 activity in the carcinoma cells and to support their survival in growth factor-deprived conditions [17]. Lahm et al [19] examined the expression of C-KIT and SCF in the cell lines of human colorectal carcinoma. They identified the presence of SCF in two cell lines and C-KIT expression in nine of 11 cell lines.

$\mathrm{Li}$ et al reported a highly activated SCF/C-KIT signaling pathway in the mucinous colorectal adenocarcinoma in the mouse model. SCF/C-KIT signaling promoted mucus secretion by activating PKC delta-MARCKS Mucin2 which upregulated MUC2 transcription [29]. Reed et al examined the expression of C-KIT in 126 colorectal carcinomas. Cytoplasmic C-KIT protein staining was seen in $1.6 \%$ of the poorly differentiated colon carcinoma. The remaining tumors revealed no detectable expression of C-KIT. The authors indicated that an SCF/C-KIT ligand autocrine loop is an important regulator of the proliferation and progression of human CRC [30].

In colorectal adenocarcinomas, C-KIT activation by SCF ligand induces signaling by different pathways including $\mathrm{PI} 3 \mathrm{~K}$, RAS, and JAK/STAT [31]. The C-KIT knockdown in CRC cells is associated with a decrease in tumor growth and colony-forming capacity [31]. Several reports indicated that C-KIT inhibitors have promising results in CRC prevention and therapy. The regorafenib (Food and Drug Administration (FDA)-approved multi-kinase inhibitor) is used for the treatment of patients with metastatic CRC with advanced disease [32].

\section{Conclusions}

To conclude, a growing body of case reports in the literature indicates the increasing incidence of coexistence of gastric GIST with CRC either synchronously or metachronously. Accordingly, in any patient with GIST, the pathologists, gastroenterologists, and surgeons should consider the possibility of coexistent a histologically different tumor. This mandates a careful preoperative workup for the proper management. Genetic counseling is an educational process that is usually conveyed by health care providers or genetic counselors. It is of utmost importance in CRC cases. This educational process provides information about the likelihood of getting cancer and helps make decisions about further genetic testing options $[33,34]$.

\section{Acknowledgments}

None to declare.

\section{Financial Disclosure}

This research did not receive any specific grant from funding agencies in the public, commercial, or not-for-profit sector.

\section{Conflict of Interest}

None to declare.

\section{Informed Consent}

Informed consent was obtained from the patient.

\section{Author Contributions}

All authors certify that they have participated sufficiently in the intellectual content and the analysis of data. Each author has reviewed the final version of the manuscript and approved it for publication. Dr. Abdullah S. Alqahtani is the principal investigator responsible for this project.

\section{Data Availability}

The authors declare that data supporting the findings of this study are available within the article.

\section{References}

1. Eisenberg BL, Pipas JM. Gastrointestinal stromal tumor-background, pathology, treatment. Hematol Oncol Clin North Am. 2012;26(6):1239-1259.

2. Mazur MT, Clark HB. Gastric stromal tumors. Reappraisal of histogenesis. Am J Surg Pathol. 1983;7(6):507-519.

3. Fonseca IB, Spitale LS, Gramatica L, Cejas H, Piccinni DJ, Ghirardi G. [Gastrointestinal stromal tumors: conceptual evolution]. Rev Fac Cien Med Univ Nac Cordoba. 2006;63(1):37-45.

4. Ramos Socarras AE, Valles Gamboa ME, Rodriguez Nunez JR, Neyra Rodriguez CM, Vargas La OF. [Gastrointestinal stroma tumor]. Rev Gastroenterol Peru. 2014;34(3):237-241.

5. Huizinga JD, Thuneberg L, Kluppel M, Malysz J, Mikkelsen HB, Bernstein A. W/kit gene required for interstitial cells of Cajal and for intestinal pacemaker activity. Nature. 1995;373(6512):347-349.

6. Hirota S, Isozaki K, Moriyama Y, Hashimoto K, Nishida $\mathrm{T}$, Ishiguro S, Kawano K, et al. Gain-of-function mutations of c-kit in human gastrointestinal stromal tumors. Science. 1998;279(5350):577-580.

7. Heinrich MC, Corless CL, Duensing A, McGreevey L, Chen CJ, Joseph N, Singer S, et al. PDGFRA activating mutations in gastrointestinal stromal tumors. Science. 2003;299(5607):708-710.

8. Vassos N, Agaimy A, Hohenberger W, Croner RS. Coexistence of gastrointestinal stromal tumours (GIST) and malignant neoplasms of different origin: prognostic im- 
plications. Int J Surg. 2014;12(5):371-377.

9. Gill AJ, Chou A, Vilain R, Clarkson A, Lui M, Jin R, Tobias $\mathrm{V}$, et al. Immunohistochemistry for SDHB divides gastrointestinal stromal tumors (GISTs) into 2 distinct types. Am J Surg Pathol. 2010;34(5):636-644.

10. Wang Y, Sun T, Sun H, Yang S, Li D, Zhou D. SCF/C-Kit/ JNK/AP-1 signaling pathway promotes claudin-3 expression in colonic epithelium and colorectal carcinoma. Int J Mol Sci. 2017;18(4):765.

11. Zhang JQ, Bosbach B, Loo JK, Vitiello GA, Zeng S, Seifert AM, Medina BD, et al. The V654A second-site KIT mutation increases tumor oncogenesis and STAT activation in a mouse model of gastrointestinal stromal tumor. Oncogene. 2020;39(49):7153-7165.

12. Garcia-Aranda M, Redondo M. Targeting receptor kinases in colorectal cancer. Cancers (Basel). 2019;11(4):433.

13. Toyota M, Hinoda Y, Takaoka A, Makiguchi Y, Takahashi T, Itoh F, Imai K, et al. Expression of c-kit and kit ligand in human colon carcinoma cells. Tumour Biol. 1993;14(5):295-302.

14. Sammarco I, Capurso G, Coppola L, Bonifazi AP, Cassetta S, Delle Fave G, Carrara A, et al. Expression of the proto-oncogene c-KIT in normal and tumor tissues from colorectal carcinoma patients. Int J Colorectal Dis. 2004;19(6):545-553.

15. Chen EC, Karl TA, Kalisky T, Gupta SK, O'Brien CA, Longacre TA, van de Rijn M, et al. KIT signaling promotes growth of colon xenograft tumors in mice and is up-regulated in a subset of human colon cancers. Gastroenterology. 2015;149(3):705-717.e702.

16. Fatrai S, van Schelven SJ, Ubink I, Govaert KM, Raats D, Koster J, Verheem A, et al. Maintenance of Clonogenic KIT(+) Human Colon Tumor Cells Requires Secretion of Stem Cell Factor by Differentiated Tumor Cells. Gastroenterology. 2015;149(3):692-704.

17. Bellone G, Carbone A, Sibona N, Bosco O, Tibaudi D, Smirne C, Martone T, et al. Aberrant activation of c-kit protects colon carcinoma cells against apoptosis and enhances their invasive potential. Cancer Res. 2001;61(5):2200-2206.

18. Zhang B, Wang J, Wang X, Zhu J, Liu Q, Shi Z, Chambers MC, et al. Proteogenomic characterization of human colon and rectal cancer. Nature. 2014;513(7518):382387.

19. Lahm H, Amstad P, Yilmaz A, Borbenyi Z, Wyniger J, Fischer JR, Suardet L, et al. Interleukin 4 down-regulates expression of c-kit and autocrine stem cell factor in human colorectal carcinoma cells. Cell Growth Differ. 1995;6(9):1111-1118.

20. Waidhauser J, Bornemann A, Trepel M, Markl B. Frequency, localization, and types of gastrointestinal stromal tumor-associated neoplasia. World J Gastroenterol. 2019;25(30):4261-4277.

21. Suzuki T, Suwa K, Hanyu K, Okamoto T, Fujita T, Yanaga $\mathrm{K}$. Large gastrointestinal stromal tumor and advanced adenocarcinoma in the rectum coexistent with an incidental prostate carcinoma: A case report. Int J Surg Case Rep. 2014;5(9):640-642.

22. Hakozaki Y, Sameshima S, Tatsuoka T, Okuyama T, Yamagata Y, Noie T, Oya M, et al. Rectal carcinoma and multiple gastrointestinal stromal tumors (GIST) of the small intestine in a patient with neurofibromatosis type 1: a case report. World J Surg Oncol. 2017;15(1):160.

23. Jafferbhoy S, Paterson H, Fineron P. Synchronous gist, colon and breast adenocarcinoma with double colonic polyp metastases. Int J Surg Case Rep. 2014;5(8):523526.

24. Markovic M, Jurisic V, Petrovic M, Dagovic A, Stankovic V, Mitrovic S. Appearance of ductal breast and colon carcinoma with gastrointestinal stromal tumor (GIST) in a female patient: an extremely rare case. Rom J Morphol Embryol. 2018;59(2):613-617.

25. Naitoh I, Okayama Y, Hirai M, Kitajima Y, Hayashi K, Okamoto T, Akita S, et al. Exophytic pedunculated gastrointestinal stromal tumor with remarkable cystic change. J Gastroenterol. 2003;38(12):1181-1184.

26. Seshadri RA, Singh SS, Ratnagiri R. Synchronous jejunal gastrointestinal stromal tumor and primary adenocarcinoma of the colon. Indian J Surg. 2012;74(2):196-198.

27. Liu S, Liu H, Dong Y, Wang F, Wang H, Chen J. Gastric carcinoma with a gastrointestinal stromal tumor - A case report and literature review. Med Sci (Paris). 2018;34(Focus issue F1):15-19.

28. Zhang P, Deng R, Xia Z, Shuai X, Chang W, Gao J, Wang $\mathrm{G}$, et al. Concurrent gastrointestinal stromal tumor and digestive tract carcinoma: a single institution experience in China. Int J Clin Exp Med. 2015;8(11):21372-21378.

29. Li G, Yang S, Shen P, Wu B, Sun T, Sun H, Ji F, et al. $\mathrm{SCF} / \mathrm{c}-\mathrm{KIT}$ signaling promotes mucus secretion of colonic goblet cells and development of mucinous colorectal adenocarcinoma. Am J Cancer Res. 2018;8(6):10641073.

30. Reed J, Ouban A, Schickor FK, Muraca P, Yeatman T, Coppola D. Immunohistochemical staining for c-Kit (CD117) is a rare event in human colorectal carcinoma. Clin Colorectal Cancer. 2002;2(2):119-122.

31. Shah YM, van den Brink GR. c-Kit as a novel potential therapeutic target in colorectal cancer. Gastroenterology. 2015;149(3):534-537.

32. Attoub S, Rivat C, Rodrigues S, Van Bocxlaer S, Bedin M, Bruyneel E, Louvet C, et al. The c-kit tyrosine kinase inhibitor STI571 for colorectal cancer therapy. Cancer Res. 2002;62(17):4879-4883.

33. Taylor KM, Kelner MJ. The emerging role of the physician in genetic counselling and testing for heritable breast, ovarian and colon cancer. CMAJ. 1996;154(8):11551158.

34. Aktan-Collan K, Haukkala A, Pylvanainen K, Jarvinen HJ, Aaltonen LA, Peltomaki P, Rantanen E, et al. Direct contact in inviting high-risk members of hereditary colon cancer families to genetic counselling and DNA testing. J Med Genet. 2007;44(11):732-738. 